\title{
Die Behandlung des schweren Schädel-Hirn- Traumas bei Kindern und Erwachsenen
}

\author{
Andreas Unterberg
}

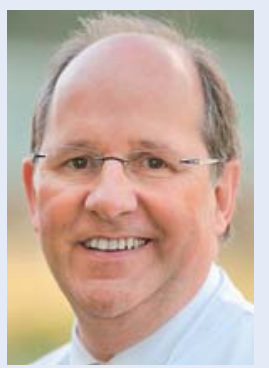

Andreas Unterberg
0

hne jeden Zweifel: In Deutschland hat sich in den letzten 10 Jahren hinsichtlich des Krankheitsbildes „Schädel-Hirn-Trauma“ vieles verändert, wenn auch nicht die Grundprinzipien bei einer Therapie.

Noch vor kurzer Zeit beherrschten junge Patienten mit Hochgeschwindigkeitstraumen (z. B. nach Verkehrsunfällen unterschiedlichster Art) das Bild vieler Intensivstationen. Durch bessere Prävention ist die Zahl dieser Patienten deutlich zurückgegangen. Stattdessen erleiden viele ältere Patienten auf der Straße oder im häuslichen Umfeld ein Schädel-Hirn-Trauma mit schwerwiegenden Folgen.

Nach Hochgeschwindigkeitstraumen dominiert oft eine diffuse Hirnschwellung den Krankheitsverlauf. Daher stehen Management und Therapie des erhöhten intrakraniellen Drucks im Vordergrund. Dies geschieht durch unterschiedliche Maßnahmen, wie moderate Hyperventilation, Oberkörperhochlagerung, Mannitol, Barbiturate und schließlich durch eine Dekompressionstrepanation. In den ersten Leitlinien der amerikanischen Brain Trauma Foundation wurde zur Behandlung des erhöhten intrakraniellen Drucks ein Stufenalgorithmus vorgeschlagen. In vielen neurotraumatologischen Zentren wurde dieser Algorithmus implementiert und angewandt. Mittlerweile ist in den weltweit akzeptierten BTF-Leitlinien die Empfehlung dieses BTF-Algorithmus zurückgenommen worden. Es liegt nämlich keine überzeugende Evidenz dafür vor, dass die Einhaltung dieses Algorithmus einer individualisierten Therapie des erhöhten intrakraniellen Drucks überlegen ist. Und so betonen die derzeit gültigen Richtlinien die Notwendigkeit der Herstellung einer normalen Physiologie, die Normalisierung des intrakraniellen Drucks sowie eines ausreichenden zerebralen Perfusionsdrucks.

Anders bei den schweren Schädel-Hirn-Traumen älterer Patienten: Diese sind vornehmlich "low velocity“Traumen und gekennzeichnet durch subdurale Blutungen und Kontusionsblutungen, oft gefördert durch eine prätraumatische Einnahme von Antikoagulantien. Bei diesen Patienten stellt sich immer wieder die Frage, ob z.B. raumfordernde Kontusionsblutungen operativ angegangen werden sollten und letztlich die Frage, inwieweit in fortgeschrittenem Lebensalter intensivste Therapiebemühungen überhaupt durchgeführt werden sollten. Denn die wichtigsten prognosebestimmenden Faktoren beim Schädel-Hirn-Trauma sind nach wie vor das Lebensalter und die Schwere des Traumas, indirekt abgeschätzt anhand der Tiefe der Bewusstlosigkeit betroffener Patienten durch den Glasgow-Coma-Score.

Vor diesem Szenario stellen die beiden Übersichtsartikel zur Behandlung des schweren Schädel-HirnTraumas bei Kindern und Erwachsenen eine wichtige Fortbildung für alle Ärzte dar, die in der Intensivmedizin tätig sind. Denn je weniger dieses Krankheitsbild behandelt wird, umso mehr geraten klinische Routinen in Vergessenheit, obwohl eine Vielzahl von Leitlinien und Empfehlungen international und national vorhanden sind. Die derzeitige Abkehr von schematischen Algorithmen macht zudem die Therapie für den noch nicht so erfahrenen Intensivmediziner schwieriger.

Wer beide Artikel zum Schädel-Hirn-Trauma liest, bemerkt die nuancierten Unterschiede, die in der Behandlung von Kindern und Erwachsenen bestehen, abgesehen von den Unterschieden in Epidemiologie und Ursachen. Wenn auch die Prinzipien der Behandlung des schweren Schädel-Hirn-Traumas im Kindesund Erwachsenenalter sich sehr ähneln, so fällt doch dieses auf: Kleinkinder haben eine signifikant schlechtere Prognose. Woran dies liegt, bleibt unklar.

In den vergangenen zwei Jahrzehnten sind eine Vielzahl von klinischen Studien zur Behandlung des schweren Schädel-Hirn-Traumas durchgeführt worden. Verschiedenste Neuroprotektiva wurden getestet, bedauerlicherweise hat sich keines als effektiv erwiesen. Aus der Meta-Analyse dieser vielfältigen Arzneimittelstudien wird nun eines immer deutlicher: Im Vordergrund der intensivmedizinischen Behandlung des schweren Schädel-Hirn-Traumas steht die Herstel- 
lung einer normalen Physiologie. Jedoch ist nicht einmal die Herstellung eines normal hohen intrakraniellen Drucks eine klar bewiesene Tatsache.

Derzeit rekrutieren drei nennenswerte internationale multizentrische Studien im Bereich des Krankheitsbildes „Schädel-Hirn-Trauma“:

1. In der Rescue-ICP-Studie wird die Effektivität der (subakut) durchgeführten Dekompressionstrepanation bei therapierefraktärer intrakranieller Hypertension geprüft.

2. Im STITCH-Trial wird die Sinnhaftigkeit der operativen Beseitigung von raumfordernden Kontusionsblutungen untersucht.

3. Im Synapse-Trial wird Progesteron als Neuroprotektivum beim schweren Schädel-Hirn-Trauma eingesetzt.
Nach wie vor ist das schwere Schädel-Hirn-Trauma lebensbedrohlich und eine Erkrankung, die zu permanenter Morbidität führen kann. Deswegen sind alle experimentellen und klinischen Bemühungen, seine Therapie zu verbessern, mehr als gerechtfertigt.

Wichtigste Strategie im Management des schweren Schädel-Hirn-Traumas ist das ständige Abwägen operativer und intensivmedizinischer Maßnahmen. Die beiden Artikel in diesem Heft reflektieren diesen interdisziplinären Ansatz beispielhaft.

\section{Korrespondenzadresse}

Prof. Dr. med. Andreas Unterberg

Neurochirurgische Klinik und Poliklinik

Universität Heidelberg

Im Neuenheimer Feld 400

69120 Heidelberg

E-Mail: Andreas.Unterberg@med.uni-heidelberg.de 